\title{
FACTORES PERSONALES DE RESILIENCIA Y AUTOCONCEPTO EN ESTUDIANTES DE PRIMARIA DE LIMA METROPOLITANA
}

\author{
Personal factors of resilience and self-concept in primary school students of \\ Metropolitan Lima \\ María Matalinares C. ${ }^{1}$, Carlos Arenas I., Juan Yaringaño L., Lidia Sotelo L., Noemí Sotelo L., \\ Gloria Díaz A., Alejandro Dioses Ch., Rodrigo Ramos C., Patricia Mendoza P., Yizza Medina R., \\ Mary Pezua V., Raúl Muratta E., Cecllia Pareja F., Rina Tipacti T. \\ Universidad Nacional Mayor de San Marcos \\ (ReCiBIDo el 05/04/2011 - AcEPtAdo el 07/07/2011)
}

\begin{abstract}
RESUMEN
La investigación tuvo como objetivo establecer la existencia o no de relación entre los factores personales de resiliencia y el autoconcepto en estudiantes de primaria de Lima Metropolitana. Se evaluó a 420 estudiantes, de ambos sexos, cuyas edades fluctuaban entre 10 y 12 años, que cursaban el quinto y sexto grado de primaria, procedentes de diferentes centros educativos estatales de Lima Metropolitana, a quienes se aplicó el inventario de Factores personales de resiliencia propuesto por Salgado (2005) y el Cuestionario de Autoconcepto Garley propuesto por Belén García, adaptado a nuestra realidad para efectos de la investigación. Los resultados mostraron que existe correlación entre los factores personales de resiliencia y autoconcepto. Se reporta una relación entre los factores autoestima y empatía con todos los componentes del autoconcepto. Al comparar el autoconcepto de varones con mujeres se encontró diferencias significativas en los componentes físico, social y familiar a favor de las mujeres. Por último, al comparar varones y mujeres en los factores de resiliencia se encontró diferencias significativas a favor de las mujeres en autoestima, empatía y resiliencia.
\end{abstract}

Palabras clave: Factores personales de resiliencia, autoconcepto, resiliencia, niños.

\begin{abstract}
The research presented was to establish whether there was any connection between the personal factors of resilience and self-concept in primary school students in Lima. For its performance was evaluated at 420 students, of both sexes, aged between 10 and 12 years, who were in the fifth and sixth grades of primary schools from different Lima state who completed the inventory of factors personal resilience proposed by Salgado (2005) and SelfConcept Questionnaire proposed by Belen García Garley, adapted to our reality for purposes of the investigation. Results showed that correlation exists between the personal factors of resilience and self-concept. He also found a relationship between the factors self-esteem and empathy with all components of the self-concept. When comparing men with women's
\end{abstract}

1 Profesora principal de la Facultad de Psicología de la UNMSM. E-mail: mmatalinaresc@unmsm.edu.pe 
self-concept were no significant differences in the physical, social and family in favor of women. Finally, when comparing men and women in the resilience factors found significant differences in favor of women in self-esteem, empathy and resilience.

Keywords: Personal factors of resilience, self-concept, resilience, children.

\section{INTRODUCCIÓN}

La resiliencia es un tema estudiado en la actualidad por la importancia que tiene para la promoción del desarrollo (Salgado, 2005). El término "resiliencia" es una castellanización de la palabra inglesa resilience, o resiliency. Este no es un término exclusivo de la psicología, y se refiere a "la capacidad de un cuerpo para recuperar su tamaño y forma original después de ser comprimido, doblado o estirado", o bien a "una capacidad para recuperarse de o ajustarse fácilmente al cambio o la mala fortuna" (Mish, 1989; citado por Kalawski y Haz, 2003).

Rutter (1993) señala que el término de resiliencia se ha definido como la capacidad de las personas para desarrollarse psicológicamente sanas y exitosas, a pesar de estar expuestas a situaciones adversas que amenazan su integridad. Rutter (1999) señala que existe una enorme variación en las respuestas de los niños para describir la resistencia a experiencias de riesgo psicosocial. Los resultados de las investigaciones para determinar la procesos que subyacen en el comportamiento resiliente subrayan los siguiente: la presencia de múltiples factores de protección frente al riesgo están involucrados; y que los niños varían en su vulnerabilidad al estrés psicosocial y la adversidad según factores genéticos o ambientales; las experiencias del ambiente familiar inciden en las diferencias individuales de los niños; la reducción de las reacciones negativas, y aumento de las positivas, influyen en la medida en que los efectos de la adversidad persisten en el tiempo; las experiencias nuevas puede proporcionar oportunidades para un "punto de inflexión" sobre los efectos; las experiencias positivas aunque en sí mismas no ejercen un gran efecto de protección pueden ser útiles para neutralizar algunos factores de riesgo; y que el procesamiento cognitivo y afectivo de experiencias puede influir en la resistencia que se desarrolla.

Badilla (2003) explica que la resiliencia es una combinación de factores que permiten a un ser humano afrontar y superar los problemas y adversidades de la vida. Por su parte, Suárez (1995) señala que la resiliencia es una combinación de factores que le permiten a un ser humano, afrontar y superar los problemas y adversidades de la vida, es decir, le permite contender con el estrés de la vida cotidiana y con las situaciones difíciles, sin que necesariamente se refiera a recuperación después de haber experimentado un trauma.

De acuerdo con Vanistendael (1997), existen cinco dimensiones de la resiliencia:

A. Existencia de redes sociales informales: la persona tiene amigos, participa de actividades con ellos y lo hace con agrado; tiene en general una buena relación con los adultos.

B. Sentido de vida, trascendencia: la persona muestra capacidad para descubrir un sentido y una coherencia en la vida.

C. Autoestima positiva: la persona se valora a sí misma, confía en sus capacidades y muestra iniciativa para emprender acciones o relaciones con otras personas porque se siente valiosa y merecedora de atención. 
D. Presencia de aptitudes y destrezas: es capaz de desarrollar sus competencias y confiar en ellas.

E. Sentido del humor: la persona es capaz de jugar, reír, gozar de las emociones positivas y de disfrutar de sus experiencias.

Benard (2002), citado por Acevedo y Mondragón (2005), indica que los estudios muestran que hay factores personales en la mayoría de personas que superan sus dificultades: competencia social, sentido de autonomía, sentido de propósito y creencia en un futuro mejor. Esto ha llevado a muchos investigadores a destacar estos atributos, creando programas de habilidades sociales y habilidades para la vida que tienden a "enseñar" las "habilidades de resiliencia". Esto permite considerar que el involucramiento de las escuelas en el proceso de promoción de la resiliencia es fundamental, dado que en diversas investigaciones se halló que fuera de la familia, el adulto que más influencia positiva puede tener sobre un niño es un profesor(a).

Ahora bien, en el Perú, Astete, Benavides y Palacios (2009) realizaron un estudio descriptivo con el objetivo de identificar el nivel de resiliencia en niños de 8 a 12 años provenientes de familias disfuncionales. El método usado fue descriptivo, de corte transversal. La muestra estuvo conformada por 30 niños asistentes al taller "Mundo al Revés" de la Casa Carrillo Maurtua que reunieron los criterios de inclusión. En la recolección utilizaron un cuestionario estructurado que aplicaron a través de una entrevista con preguntas dicotómicas. El instrumento se aplicó de forma individual, previa firma del asentimiento informado de los padres. Los resultados mostraron que los niños presentan un alto nivel de resiliencia (73.3 por ciento), según los componentes resilientes: competencia social, resolución de problemas y autonomía y sentido de propósito y de futuro.

Flores (2008) investiga la resiliencia y el proyecto de vida. El objetivo fundamental de esta investigación era determinar si existe relación entre los niveles de resiliencia y el grado de definición del proyecto de vida en estudiantes del tercer año de secundaria de colegios nacionales y particulares de la UGEL 03. La técnica de muestreo fue no probabilística, seleccionó una muestra de estudiantes de ambos sexos (Varones igual a 200; Mujeres igual a 200). La investigación es de tipo descriptivo correlacional, se emplearon como instrumentos de investigación la Escala de Resiliencia de Wagnild y Young (1993) y la Escala para la Evaluación del Proyecto de Vida de García (2002). Los resultados mostraron que existe relación altamente significativa $(\mathrm{p}<0,0001)$ entre el grado de resiliencia y el grado de definición del proyecto de vida, tanto en la muestra de estudiantes varones como en la de mujeres.

Aguirre (2002) estudia la capacidad y factores asociados a la resiliencia, en adolescentes del CE Mariscal Andrés A. Cáceres, del sector IV de Pamplona Alta S.J.M. En este estudio de tipo descriptivo exploratorio de corte transversal se aplicó la Lista de Chequeo de Goldstein modificado para determinar la capacidad de resiliencia (C.R) y un formulario para identificar los factores asociados a la resiliencia (F.A.R.) en 214 adolescentes del mencionado C.E. Se obtuvo que el $97.6 \%$ poseen una C.R entre alta y media, de los cuales en el $54.3 \%$ se presentan más factores protectores (F.P) y en el $44.4 \%$ se presentan más factores de riesgo (F.R). En 24.3\% la familia es considerada como el F.P más importante, $20.6 \%$ es la comunidad y $10.75 \%$ consideran a la escuela como F.P. Entre los adolescentes con C.R alta, el $20.5 \%$ lo constituyen las mujeres y el $14 \%$ los varones. 
Ahora bien, respecto del autoconcepto es necesario señalar que la mayoría de los autores interpretan el autoconcepto globalmente como conjunto integrado de factores o actitudes relativos al yo, señalando básicamente tres: cognitivos (pensamientos), afectivos (sentimientos) y conativos (comportamientos); que, de considerarlos individualmente podrían identificarse de la siguiente manera: el primer factor como autoconcepto propiamente dicho, el segundo como autoestima y el tercero como autoeficacia.

De acuerdo con Loperena (2008), habría que establecer una diferencia entre estos términos, limitando el autoconcepto a los aspectos descriptivos o cognoscitivos del self y la autoestima para designar los aspectos evaluativos y afectivos. Actualmente, la tendencia más generalizada es la de integrar en el autoconcepto elementos cognitivo-afectivos, puesto que ni teórica ni empíricamente resulta factible su disociación debido a que toda percepción de sí mismo lleva implícita una valoración afectiva, esto no podría ser de otra manera, ya que los elementos subjetivos que implican un juicio de valor están mediados por la propia subjetividad del sujeto.

De este modo, para Musitu, García y Gutiérrez (1997) el autoconcepto puede entenderse como la percepción que el individuo tiene de sí mismo, basado en sus experiencias con los demás y en las atribuciones de su propia conducta. Involucra componentes emocionales, sociales, físicos y académicos. Se trata de una configuración organizada de percepciones de sí mismo, admisible a la conciencia y al conocimiento. Es un esquema cognitivo muy complejo construido a través de experiencias previas con respecto al mundo que lo rodea.

Epstein (1981) dice que es una realidad compleja, integrada por diversos autoconceptos más concretos, como el físico, social, emocional y académico y los caracteriza como una realidad dinámica que se modifica con la experiencia; se desarrolla a partir de las experiencias sociales, especialmente con las personas significativas; es la clave para la comprensión de los pensamientos, sentimientos y conductas o comportamientos de las personas. La autoestima, como componente afectivo del autoconcepto, es uno de los factores más importantes, si no el que más, de los que rigen el comportamiento humano, de ahí su importancia en la vida escolar. Un niño con inteligencia superior a la media y con poca autoestima puede ir "a las justas", mientras que otro de inteligencia media pero con mucha autoestima puede obtener buenos resultados.

En el Perú, Mori (1989) examinó las posibles relaciones entre condición socio-económica y sexo y los cinco dominios específicos y la autovaloración global del autoconcepto. Se aplicó la prueba de Autoconcepto de Harter (1985) a una muestra de 210 niños de 11 a 12 años que cursaban el sexto grado de primaria: 108 de condición socio-económica baja (44 niños y 58 niñas). Los resultados apoyan parcialmente las hipótesis, encontrándose sólo diferencias significativas entre autoconcepto y condición socio-económica en el dominio específico de aceptación social. Las relaciones de autoconcepto y sexo fueron significativas para los dominios específicos aceptación social, desempeño atlético y autovaloración global. Los hallazgos sugieren que el dominio específico de aceptación social es el más afectado por la variable socio-económica. Los efectos más sistemáticos observados con la variable sexo en los dominios específicos de aceptación social, desempeño atlético y autovaloración global podrían atribuirse a las estrategias diferenciales en la socialización de niños y niñas. 
Pazzaglia (1990) aplicó la versión castellana del Inventario de Autoestima creado por Barksdale (1978) y el Inventario de Preferencias Personales de Edwards, a un grupo de 100 alumnos de uno y otro sexo del cuarto año de secundaria del Colegio Parroquial "Fe y Alegría 5", ubicado en San Juan de Lurigancho. Previamente se determinó la validez y confiabilidad del Inventario de Autoestima en 100 alumnos de características similares a los de la muestra estudiada. Los resultados del estudio no indican relación entre el Autoconcepto y las Preferencias Personales en el sexo femenino; sin embargo, en el sexo masculino se evidencia únicamente relación entre el Autoconcepto y una de las variables de Preferencias Personales: la de Rendimiento.

Aguirre (1994) realizó un estudio con 76 niños desplazados de diversas zonas del Perú que fueron víctimas de violencia subversiva y que son residentes en una Comunidad Rural de Lima. Se determinó el nivel de Autoconcepto, el nivel de las dimensiones de Personalidad y la relación existente entre ambas variable. Se aplicó la Escala de Autoconcepto para Niños de Mc. Daniel y Piers y el Inventario de Personalidad de Eysenck para Niños (JEPI); se validó los instrumentos para dicha población, obteniéndose 0.62 y 0.68 , para cada uno de los instrumentos. Los resultados mostraron que los niños tenían un nivel bajo de autoconcepto, que tendían a la introversión y a la inestabilidad emocional. Se encontró relación significativa entre la variable Extroversión con el Área Social de la Escala de Autoconcepto General. La variable Neuroticismo, mostró una relación estadísticamente significativa con el Autoconcepto General y el Área Emocional. Además, se encontraron otras relaciones con las variables de Tiempo de Residencia en la ciudad de Lima y Lugar de Nacimiento relacionándose estadísticamente con la Dimensión Neuroticismo y el Autoconcepto, respectivamente.

Caravedo (2001) realizó una investigación descriptiva sobre el autoconcepto de hijos de padres separados, mediante la aplicación de la Escala de Autoconcepto para niños Mc. Daniel-Piers. Con la finalidad de contribuir y realmente enfatizar en la importancia que tiene la estructura familiar para el niño, en el desarrollo de este proceso para su futuro emocional, social e intelectual. La investigación se realizó con niñas entre 10 y 12 años de edad, que se ubicaron entre quinto y sexto de primaria de un Colegio Particular de Lima Metropolitana. Los resultados obtenidos reflejan en el análisis general que el autoconcepto de los hijos de padres separados tiende a ser más negativo que el autoconcepto de hijos de familias estructuradas o donde los padres viven juntos.

Orbegoso (2002) estudia de manera comparativa el autoconcepto y la depresión en niños obesos y no obesos. En una muestra conformada por 120 niños provenientes del Hospital Nacional, clasificados en dos grupos: un grupo de 60 niños (grupo I) y otro de no obesos (grupo II). La edad promedio de los niños fue de 8 y 13.9 años. El objetivo de estudio ha sido comparar el autoconcepto y depresión en niños obesos y no obesos. Se utilizó la Escala d Autoconcepto para niños de Mc. Daniel-Piers y el Inventario de Depresión para niños de M. Kovacs (CDI). La condición de obesidad fue definida como el índice de masa corporal (IMC) de 95, utilizando los criterios señalados por la (OMS), respectivamente. Los resultados no mostraron diferencias estadísticamente significativas entre obesidad y autoconcepto $(\mathrm{p}=0.445)$ ni entre obesidad y depresión $(\mathrm{p}=0.402)$. No se encontró significación en la interacción de la condición de obesidad con el sexo respecto al autoconcepto y a la depresión, similares resultados se obtuvieron con la edad. Se encontró una diferencia significativa, entre niños obesos y no obesos en la sub-área académico del 
área del rendimiento de la Escala de Autoconcepto. En conclusión, no se encontró una evidencia que permita establecer que la obesidad influye en el autoconcepto y depresión.

Suxe (2004) realiza una investigación aplicada de tipo descriptivo correlacional, tiene como objetivo central, determinar el grado de relación entre las actitudes maternas de aceptación, sobreprotección, sobreindulgencia y rechazo y el autoconcepto de los niños, y al mismo tiempo identificar, si variables demográficas como sexo del niño, edad, orden de nacimiento, número de hijos de las madres, grado de instrucción y ocupación pueden predecir un autoconcepto negativo. Los participantes fueron seleccionados de manera intencional, y estuvieron conformados por 113 niños de ambos sexos, de 6 y 7 años de edad, de un colegio nacional de San Martín de Porres y 112 madres cuyos hijos fueron evaluados. Se aplicaron como instrumentos de evaluación la Escala de Autoconcepto de Mc Daniel Piers y la Escala Adaptada de la Relación Madre-Niño de Roth. Entre los resultados obtenidos en esta investigación se encontró que las actitudes de aceptación y rechazo de las madres predicen un autoconcepto general positivo o negativo en los niños. En el área de autoconcepto social se encontró que cuando las madres muestran aceptación por sus hijos el autoconcepto social negativo disminuye, cuando el hijo es de sexo masculino el autoconcepto es negativo, y cuando la madre tiene menor grado de instrucción el autoconcepto social negativo de su hijo es mayor. En el autoconcepto emocional se obtuvo que cuando el hijo es de sexo masculino el autoconcepto emocional es negativo. En el autoconcepto de rendimiento se observó que el ser mayor la edad del niño el autoconcepto de rendimiento negativo es menor, al ser el hijo último, el autoconcepto de rendimiento negativo es mayor $\mathrm{y}$ al incrementarse las conductas de rechazo de la madre el autoconcepto de rendimiento se vuelve más negativo. En el autoconcepto de autopercepción (fortaleza y apariencia física) se encontró que las niñas muestran un autoconcepto negativo.

Mori (2002) investiga con el propósito de construir un modelo explicativo de las múltiples relaciones que pueden establecerse entre algunos dominios del autoconcepto, algunas dimensiones de la personalidad, ciertos aspectos de la percepción del compromiso parental en las actividades educativas, tanto en el hogar como en la escuela y el rendimiento académico. La muestra estuvo constituida por 473 estudiantes del sexto grado de educación primaria, hombres y mujeres pertenecientes a los grupos socioeconómicos no extremos (B, C, D). El $53 \%$ fueron varones y el $46.7 \%$ mujeres. Las edades variaban de 10 a 13 años. Los instrumentos de investigación utilizados fueron: el Cuestionario de Personalidad de Eysenck EPQ-J para niños, el Perfil de Autopercepción (Autoconcepto) de Susan Harter para niños, el Cuestionario de Percepción en los Niños del Compromiso Parental con su Educación, la Prueba de Rendimiento en Comunicación Integral y Lógico-Matemática. Una de las conclusiones más importantes de este estudio hace referencia al Modelo de múltiples relaciones causales especificado por las 5 ecuaciones estructurales planteadas que se ajusta adecuadamente para los alumnos del sexto grado de educación primaria. Este modelo sostiene que el Rendimiento escolar está influenciado por los Dominios del autoconcepto en las dimensiones de Desempeño escolar y Autovaloración Global, por las percepciones del compromiso parental basado en la Escuela y el Hogar y las características de Personalidad como son el Neuroticismo y Extroversión. Pero de manera simultánea explica que la percepción de la Dimensión de Autovaloración Global es a su vez influenciada por la percepción de la Dimensión de Desempeño escolar, el propio 
rendimiento y las características de Personalidad como son el Neuroticismo y Extroversión. También la percepción de la Dimensión de Desempeño escolar está influenciada por el propio rendimiento y las características de Personalidad. Por último las características de personalidad son influyentes en la percepción del compromiso parental basado en la escuela y junto con esta en la percepción del compromiso parental basado en el hogar.

\section{OBJETIVOS}

\section{Objetivo general}

Relacionar los factores personales de resiliencia con el autoconcepto de los estudiantes de primaria de Lima metropolitana.

\section{Objetivos específicos}

6. Adaptar y baremar el Cuestionario de Autoconcepto propuesto por Belén García Torres.

7. Diagnosticar los Factores personales de resiliencia de los estudiantes de primaria de Lima Metropolitana.

8. Diagnosticar las dimensiones del autoconcepto de los estudiantes de primaria de Lima Metropolitana.

9. Comparar los Factores personales de resiliencia de los estudiantes de primaria de Lima Metropolitana según la variable sexo.

10. Comparar las dimensiones del autoconcepto de los estudiantes de primaria de Lima Metropolitana según la variable sexo.

\section{Hipótesis}

Los niños que presentan mayor nivel en los factores personales de resiliencia tienden a presentar también un mayor nivel de autoconcepto.

\section{MÉTODO}

Para la realización de la presente investigación se empleo el método descriptivo con un diseño correlacional y comparativo. Se evaluó a 420 estudiantes de $5 .{ }^{\text {to }}$ y $6 .{ }^{\text {to }}$ de primaria, de ambos sexos, cuyas edades fluctúan entre los 10 y 12 años procedentes de centros educativos estatales de Lima Metropolitana. Dado el carácter de los variables a estudiar se utilizó la técnica psicométrica y de observación indirecta.

\section{INSTRUMENTOS}

\section{El inventario de Factores personales de resiliencia}

Diseñada por Ana Cecilia salgado (2004) en base a la propuesta de resiliencia de la Regional Training (1999), la prueba mide los factores personales de la resiliencia. El inventario consta 
de cinco factores: la autoestima, la empatía, la autonomía, el humor y la creatividad. La prueba consta de 48 afirmaciones y el sujeto examinado tiene dos opciones de respuesta: Sí y No. La administración es en forma colectiva en un tiempo aproximado de 30 minutos.

El instrumento tiene validez de contenido por criterio de jueces. La confiabilidad fue realizada a través de tres tipos de análisis: el primero observando el monto de valores perdidos para las variables; luego se presenta la información descriptiva básica en base a medidas de tendencia central, de dispersión y de distribución; luego un examen de diferencias entre variables demográficas a través del ANOVA de dos vías 2x4, en que las variables fueron género y edad, que fueron examinadas independientemente $y$ en interacción sobre los puntajes de los factores de resiliencia. El estudio que se presenta realizó análisis adicionales de confiabilidad para garantizar la confiabilidad de la prueba.

\section{El Cuestionario de Autoconcepto de Garley}

Propuesto por Belén García Torres, el test consta de seis dimensiones: física, social, intelectual, familiar, sensación de control y personal. La primera dimensión evalúa el grado de aceptación y satisfacción con el propio aspecto o apariencia física; la segunda indica cómo se percibe el sujeto en cuanto a su habilidad para ser aceptado por los otros y para tener amigos. La dimensión intelectual nos muestra cómo se evalúa el sujeto respecto a sus capacidades intelectuales y a su rendimiento académico. La dimensión familiar evalúa cómo se relaciona el sujeto con su familia y el grado de satisfacción que le proporciona su situación familiar. La dimensión sensación de control es la dimensión más novedosa el sujeto valora en el plano de los objetos, de las personas y del pensamiento en qué grado cree que controla esa realidad. Por último, la dimensión personal es una valoración global como persona. La prueba consta de 48 afirmaciones y el sujeto examinado tiene cinco opciones de respuesta: nunca, pocas veces, no sabría decir, muchas veces y siempre. La administración es en forma colectiva en un tiempo aproximado de 30 minutos. La prueba tiene confiabilidad y validez para la población española. Se ha realizado el proceso de adaptación y baremación a nuestro medio.

\section{RESULTADOS}

\section{Análisis descriptivo}

A continuación se realiza el análisis descriptivo de los datos obtenidos en base a los estadísticos de tendencia central (media, moda, mediana, desviación típica y varianza)

TABLA 1. Resultados descriptivos en los factores personales de resiliencia

\begin{tabular}{lcccccc}
\hline & Autoestima & Empatía & Autonomía & Humor & Creatividad & Resiliencia \\
\hline N. & 420 & 420 & 420 & 420 & 420 & 420 \\
Media & 7,70 & 6,78 & 6,39 & 5,59 & 4,61 & 31,07 \\
Nivel & Medio & Medio & Medio & Medio & Medio & Promedio \\
Desv. Típ. & 1,518 & 1,569 & 1,712 & 1,607 & 1,380 & 4,972 \\
\hline
\end{tabular}


De acuerdo con los resultados de la Tabla 1, se observa que el promedio de las puntuaciones obtenidas por los estudiantes, en cada uno de los factores personales de Resiliencia se encuentran dentro de un nivel medio (Salgado, 2004). Siendo la media de Autoestima, 7,70; Empatía, 6,78; Autonomía, 6,39; Humor, 4,61; y Creatividad, 4,61. En el caso de la Resiliencia total se observa una media de 31,07 que corresponde a un nivel promedio y una desviación típica es 4,972.

TABLA 2. Resultados descriptivos en las dimensiones de autoconcepto

\begin{tabular}{lcccccc}
\hline & Físico & Social & Familiar & Intelectual & Personal & Control \\
\hline $\mathrm{N}$ & 420 & 420 & 420 & 420 & 420 & 420 \\
Media & 29,60 & 28,08 & 30,81 & 27,72 & 28,28 & 26,21 \\
Desv. típ. & 6,795 & 5,919 & 5,579 & 6,364 & 4,899 & 5,232 \\
\hline
\end{tabular}

De acuerdo con los resultados de la Tabla 2, se observa que el promedio de la muestra en las dimensiones de autoconcepto se encuentra dentro de un nivel medio (según Salgado 2004). Siendo la media de Autoconcepto Físico, 29,60; Aceptación Social, 28,08; Autoconcepto Familiar, 30,81; Autoconcepto Intelectual, 27,72; Autoconcepto Personal, 28,28; y Sensación de Control, 26,21.

TABLA 3. Resultados porcentuales en los factores personales de resiliencia, según nivel

\begin{tabular}{lcccccccccc}
\hline & \multicolumn{2}{c}{ Autoestima } & \multicolumn{2}{c}{ Empatía } & \multicolumn{2}{c}{ Autonomía } & \multicolumn{2}{c}{ Humor } & \multicolumn{2}{c}{ Creatividad } \\
\hline Nivel & $\mathrm{F}$ & $\%$ & $\mathrm{f}$ & $\%$ & $\mathrm{f}$ & $\%$ & $\mathrm{f}$ & $\%$ & $\mathrm{f}$ & $\%$ \\
\hline Bajo & 35 & 8,3 & 28 & 6,7 & 21 & 5,0 & 44 & 10,5 & 36 & 8,6 \\
Medio & 251 & 59,8 & 243 & 57,9 & 274 & 65,2 & 262 & 62,4 & 272 & 64,8 \\
Alto & 134 & 31,9 & 149 & 35,5 & 125 & 29,8 & 114 & 27,1 & 112 & 26,7 \\
\hline Total & 420 & 100,0 & 420 & 100,0 & 420 & 100,0 & 420 & 100,0 & 420 & 100,0 \\
\hline
\end{tabular}

Según la Tabla 3, los alumnos del quinto y sexto grado de educación primaria presentan en mayor porcentaje en el nivel medio en todos los factores personales de resiliencia. Autoestima 59,8\% (Nivel Medio), Empatía 57,9\% (Nivel Medio), Autonomía 565,2\% (Nivel Medio), Humor 62,4\% (Nivel Medio) y Creatividad 69,8\% (Nivel Medio). 


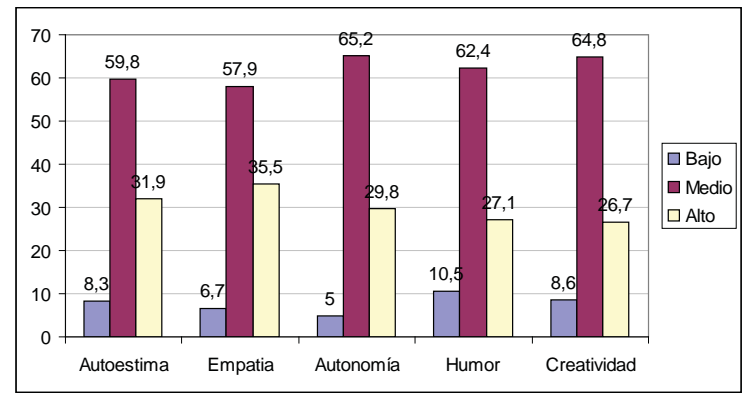

FIGURA 1. Resultados porcentuales en los factores personales de resiliencia, según nivel.

De acuerdo con la Figura 1, se observa que los alumnos del quinto y sexto grado de educación primaria, tienen el mayor porcentaje de nivel medio en factor personal Autonomía $(57,9 \%)$ en relación al resto de factores personales.

TABLA 4. Resultados porcentuales en resiliencia, según nivel

\begin{tabular}{lcc}
\hline & \multicolumn{2}{c}{ Resiliencia } \\
\hline Nivel & F & $\%$ \\
\hline Muy Bajo & 2 & 0,5 \\
Bajo & 25 & 6,0 \\
Medio & 254 & 60,5 \\
Alto & 136 & 32,4 \\
Muy Alto & 3 & 0,7 \\
\hline
\end{tabular}

Según la Tabla 4, los alumnos del quinto y sexto grado de educación primaria presentan en el nivel Muy Bajo, 0,5\%; nivel Bajo de resiliencia, 6,0\%; nivel medio de resiliencia, 60,5; nivel Alto de resiliencia, 32,4\%, y nivel Muy Alto de resiliencia 0,7\%.

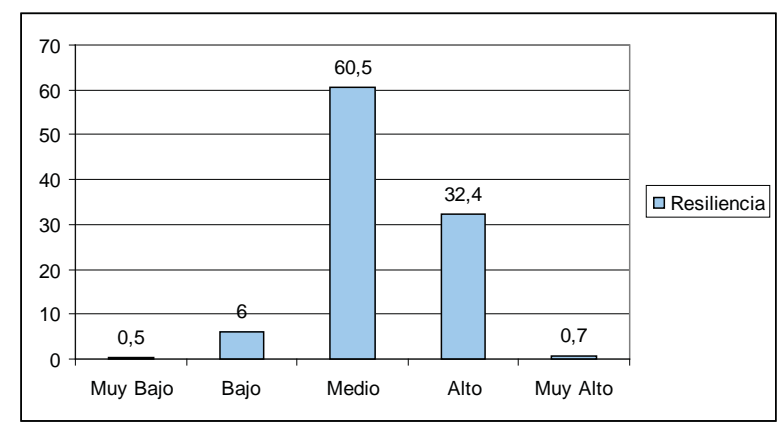

FIGURA 2. Resultados porcentuales en resiliencia, según nivel. 
De acuerdo con la Figura 2, se observa que los alumnos del quinto y sexto grado de educación primaria, tienen el mayor porcentaje de nivel medio resiliencia $(60,5 \%)$.

\section{Análisis Psicométrico}

Para el análisis psicométrico del Cuestionario de Autoconcepto Garley, se analizó la validez de construcción, mediante el estudio de la estructura interna de la prueba, para ello se procedió a organizar la matriz de correlaciones entre los ítems y las dimensiones empleándose para ello la correlación de Pearson. En el caso de la confiabilidad se optó por el método que implica una sola aplicación del instrumento, obteniéndose la consistencia interna mediante el coeficiente alfa de Cronbach.

\section{Validez del Cuestionario de Autoconcepto Garley}

En la Tabla 5 se muestran los porcentajes de varianza explicada y del porcentaje de varianza que representa cada uno de ellos. Los autovalores expresan la cantidad de la varianza total que está explicada por cada factor; y los porcentajes de varianza explicada asociados a cada factor se obtienen dividiendo su correspondiente autovalor por la suma de los autovalores (la cual coincide con el número de variables). Por defecto, se extraen tantos factores como autovalores mayores que 1 tiene la matriz analizada.

TABLA 5. Varianza total explicada de las dimensiones del autoconcepto

\begin{tabular}{|c|c|c|c|c|c|c|}
\hline \multirow{2}{*}{ Componente } & \multicolumn{3}{|c|}{ Autovalores iniciales } & \multicolumn{3}{|c|}{$\begin{array}{l}\text { Sumas de las saturaciones al } \\
\text { cuadrado de la extracción }\end{array}$} \\
\hline & Total & $\begin{array}{l}\% \text { de la } \\
\text { varianza }\end{array}$ & $\begin{array}{c}\% \\
\text { acumulado }\end{array}$ & Total & $\begin{array}{l}\% \text { de la } \\
\text { varianza }\end{array}$ & $\begin{array}{c}\% \\
\text { acumulado }\end{array}$ \\
\hline 1 & 3,230 & 53,836 & 53,836 & 3,230 & 53,836 & 53,836 \\
\hline 2 & ,779 & 12,982 & 66,818 & & & \\
\hline 3 & ,645 & 10,746 & 77,565 & & & \\
\hline 4 &, 511 & 8,511 & 86,076 & & & \\
\hline 5 & ,452 & 7,528 & 93,604 & & & \\
\hline 6 & ,384 & 6,396 & 100,000 & & & \\
\hline
\end{tabular}

En la Tabla 5, se observa que hay 1 autovalores mayor que 1, por lo que el procedimiento extrae 1 factor que consigue explicar un 53,836\% de la varianza de los datos originales. La tabla muestra también, para este factor con autovalor mayor que 1, la suma de las saturaciones al cuadrado. Este resultado coincide con la estructura del Cuestionario pues todas las dimensiones (6) son parte un único factor: Autoconcepto. 
A continuación se encuentra la solución factorial propiamente dicha. Contiene las correlaciones entre las variables originales (o saturaciones) y cada uno de los factores.

TABLA 6. Matriz de componentes de las dimensiones del autoconcepto

\begin{tabular}{lc}
\hline & Componente \\
\cline { 2 - 2 } & 1 \\
\hline Físico &, 805 \\
Social &, 700 \\
Familiar &, 712 \\
Intelectual &, 753 \\
Personal &, 740 \\
Control &, 686 \\
\hline
\end{tabular}

\section{Método de extracción: Análisis de componentes principales}

De acuerdo con la Tabla 6, al comparar las saturaciones relativas de cada dimensión en el único factor, podemos apreciar que está constituido por las dimensiones Autoconcepto Físico (0,805); Aceptación Social (0,700); Autoconcepto Familiar (0,712); Autoconcepto Intelectual $(0,753)$; Autoconcepto Personal $(0,740)$; y Sensación de Control $(0,686)$. Todas estas variables saturan en un único factor porque constituyen un grupo diferenciado de variables dentro de la matriz de correlaciones. Este factor es el autoconcepto según la propuesta de la autora del test.

\section{Confiabilidad del Cuestionario de Autoconcepto Garley}

El Alpha de Cronbach calculado para el cuestionario alcanza un valor de 0,877 de manera que se puede decir que el cuestionario de autoconcepto de Garley nos permite obtener puntajes confiables.

TABLA 7. Estadísticos de fiabilidad del Cuestionario de Autoconcepto Garley

\begin{tabular}{cc}
\hline Alfa de Cronbach & N. ${ }^{\circ}$ de elementos \\
\hline, 877 & 48 \\
\hline
\end{tabular}

Al analizar la validez de los ítems se tuvieron en cuenta dos criterios: en primer lugar, que la correlación ítem-test corregido fuera mayor de 0.20; y en segundo lugar, que la presencia del ítem, a pesar de que pudiera tener una correlación ítem-test corregido inferior a 0.20 , incrementara el coeficiente alfa de Cronbach. 
Las correlaciones ítem-test oscilan entre -0,052 y 0,565, observándose que los ítems 16 , 17, 20, 24, 36 y 39 no obtienen correlaciones significativas. En especial el ítem 36 ("Puedo conseguir que otros hagan lo que yo quiero.") presentan una correlación negativa $(-0,052)$ y el alfa de Cronbach sube a 0,880 si se elimina el ítem. De acuerdo con estos resultados se decide eliminar los ítems 17, 20 y 36 de la versión final.

TABLA 8. Estadísticos de ítems del Cuestionario de Autoconcepto Garley

\begin{tabular}{|c|c|c|c|}
\hline Ítem & $\begin{array}{c}\text { Media si se } \\
\text { elimina } \\
\text { el ítem }\end{array}$ & $\begin{array}{c}\text { Correlación } \\
\text { Ítem-test } \\
\text { corregida }\end{array}$ & $\begin{array}{c}\text { Alfa de } \\
\text { Cronbach } \\
\text { si se elimina } \\
\text { ítem }\end{array}$ \\
\hline Item1 & 170,77 & ,422 &, 873 \\
\hline item2 & 170,46 & ,376 & , 874 \\
\hline item3 & 170,32 & ,249 & , 876 \\
\hline item4 & 170,87 & ,395 & , 873 \\
\hline item5 & 170,29 & ,428 &, 873 \\
\hline item6 & 171,18 & ,281 & ,875 \\
\hline item7 & 170,59 & ,455 & ,873 \\
\hline item8 & 170,66 & ,208 & , 876 \\
\hline item9 & 169,96 & ,377 & ,874 \\
\hline item10 & 170,60 & ,497 & ,872 \\
\hline item11 & 170,58 & ,289 & ,875 \\
\hline item12 & 170,33 & ,358 & ,874 \\
\hline item13 & 170,65 & ,460 & ,872 \\
\hline item14 & 170,80 & ,266 & ,875 \\
\hline item 15 & 170,46 & ,392 & ,873 \\
\hline item16 & 170,38 & ,197 & ,876 \\
\hline item17 & 170,53 & ,116 & ,878 \\
\hline item18 & 171,26 & ,368 & ,874 \\
\hline item19 & 170,92 & ,451 &, 873 \\
\hline item 20 & 170,70 & 135 & ,878 \\
\hline item 21 & 171,63 & ,293 & ,875 \\
\hline item 22 & 170,84 &, 355 & ,874 \\
\hline item 23 & 169,99 &, 341 & ,874 \\
\hline item 24 & 171,22 & ,193 & ,877 \\
\hline
\end{tabular}

\begin{tabular}{|c|c|c|c|}
\hline Ítem & $\begin{array}{l}\text { Media si se } \\
\text { elimina } \\
\text { el ítem }\end{array}$ & $\begin{array}{c}\text { Correlación } \\
\text { Ítem-test } \\
\text { corregida }\end{array}$ & $\begin{array}{c}\text { Alfa de } \\
\text { Cronbach } \\
\text { si se elimina } \\
\text { ítem }\end{array}$ \\
\hline Item 25 & 170,69 &, 565 &, 871 \\
\hline Item 26 & 171,43 & ,313 & ,875 \\
\hline Item 27 & 170,57 & ,376 & ,874 \\
\hline Item 28 & 170,92 & ,363 & 874 \\
\hline item 29 & 170,12 & ,468 & ,872 \\
\hline item30 & 170,88 & ,311 & ,875 \\
\hline item31 & 170,14 & ,368 & ,874 \\
\hline item32 & 170,53 & ,333 &, 874 \\
\hline item33 & 170,16 & ,347 & ,874 \\
\hline item34 & 171,27 & ,386 & ,874 \\
\hline item35 & 170,70 & ,428 & ,873 \\
\hline item36 & 172,19 &,- 052 &, 880 \\
\hline item37 & 170,52 &, 440 &, 873 \\
\hline item38 & 170,73 & ,484 & ,872 \\
\hline item39 & 170,58 & , 146 & ,877 \\
\hline item 40 & 171,07 & ,394 & ,873 \\
\hline item41 & 170,19 & ,275 & ,875 \\
\hline item42 & 171,38 & ,302 & ,875 \\
\hline item43 & 170,81 & ,415 &, 873 \\
\hline item44 & 171,28 & ,279 &, 875 \\
\hline item45 & 170,19 & ,218 & ,876 \\
\hline item 46 & 171,01 & ,445 & ,872 \\
\hline item47 & 170,37 & ,434 & ,873 \\
\hline item 48 & 170,04 & ,241 & , 876 \\
\hline
\end{tabular}

Según los resultados obtenidos, el Cuestionario de Autoconcepto Garley, tiene validez de construcción, por lo tanto el constructo psicológico autoconcepto es valido en nuestro medio. Además tiene confiabilidad por consistencia interna.

\section{Constrate de normalidad de datos}

En la Tabla 9 se muestran la prueba de Bondad de Ajuste a la curva normal de Kolmogorov-Smirnov de los puntajes obtenidos en rendimiento académico en Ciencia, Tecnología y Ambiente, así como en Estilos de aprendizaje. Este tipo de análisis estadístico permite contrastar la significación de los puntajes obtenidos con el ajuste a la distribución normal. 
TABLA 9. Prueba de Normalidad

\begin{tabular}{lcccccc}
\hline & \multicolumn{2}{c}{ Kolmogorov-Smirnov $^{\mathrm{a}}$} & \multicolumn{3}{c}{ Shapiro-Wilk } \\
\hline FísICO & Estadístico & $\mathrm{gl}$ & Sig. & Estadístico & $\mathrm{gl}$ & Sig. \\
SOCIAL &, 069 & 420 &, 000 &, 971 & 420 &, 000 \\
FAMILIAR &, 050 & 420 &, 014 &, 990 & 420 &, 006 \\
INTELECTUAL &, 089 & 420 &, 000 &, 974 & 420 &, 000 \\
PERSONAL &, 081 & 420 &, 000 &, 975 & 420 &, 000 \\
CONTROL &, 120 & 420 &, 000 &, 938 & 420 &, 000 \\
autoestima &, 192 & 420 &, 000 &, 933 & 420 &, 000 \\
empatía &, 136 & 420 &, 000 &, 958 & 420 &, 000 \\
autonomía &, 125 & 420 &, 000 &, 963 & 420 &, 000 \\
humor &, 131 & 420 &, 000 &, 962 & 420 &, 000 \\
creatividad &, 179 & 420 &, 000 &, 946 & 420 &, 000 \\
resiliencia &, 053 & 420 &, 006 &, 994 & 420 &, 069 \\
\hline
\end{tabular}

$* \mathrm{p}>0,05$

a. Corrección de la significación de Lilliefors

De acuerdo con la Tabla 9, se observa que solo los puntajes alcanzados por los sujetos en las dimensiones de autoconcepto y factores personales de resiliencia presentan índices significativos menores a 0,05 (Sig. <0,05), lo que significa que los datos no se asemejan a una distribución normal.

De acuerdo con los resultados de la Prueba de Kolmogorov-Smirnov con corrección de la significación de Lilliefors, se rechaza la hipótesis de normalidad de los datos, por lo tanto se decide aplicar pruebas no paramétricas para el contraste de las hipótesis. 


\section{Análisis Inferencial}

TABLA 10. Relación entre factores de resiliencia y dimensiones de autoconcepto

\begin{tabular}{|c|c|c|c|c|c|c|c|}
\hline & & Autoestima & Empatia & Autonomía & Humor & Creatividad & Resiliencia \\
\hline \multirow{3}{*}{ Físico } & Rho spearman &, $211^{* * *}$ &, $144^{* *}$ &, 060 &, 056 & ,090 &, $183^{* *}$ \\
\hline & Sig. &, 000 & ,003 & ,223 & ,249 & ,066 &, 000 \\
\hline & $\mathrm{N}$ & 420 & 420 & 420 & 420 & 420 & 420 \\
\hline \multirow{3}{*}{ Social } & Rho spearman &, $263^{* *}$ &, $194^{* *}$ &, 090 & $136^{* *}$ &, $119^{*}$ &, $249^{* *}$ \\
\hline & Sig. &, 000 &, 000 & ,066 & ,005 & ,015 &, 000 \\
\hline & $\mathrm{N}$ & 420 & 420 & 420 & 420 & 420 & 420 \\
\hline \multirow{3}{*}{ Familiar } & Rho spearman &, $283^{* *}$ &, $218^{* *}$ &, $164^{* *}$ &, $220^{* *}$ &, $168^{* *}$ &, $341^{\text {*** }}$ \\
\hline & Sig. &, 000 &, 000 &, 001 &, 000 &, 001 &, 000 \\
\hline & $\mathrm{N}$ & 420 & 420 & 420 & 420 & 420 & 420 \\
\hline \multirow{3}{*}{ Intelectual } & Rho spearman &, $221^{* * *}$ &, $197^{* *}$ &, $127^{* *}$ &, $163^{* *}$ &, $155^{* *}$ & $272^{* *}$ \\
\hline & Sig. &, 000 &, 000 & ,009 & ,001 &, 001 &, 000 \\
\hline & $\mathrm{N}$ & 420 & 420 & 420 & 420 & 420 & 420 \\
\hline \multirow{3}{*}{ Personal } & Rho spearman &, $215^{* *}$ &, $182^{* *}$ &, $145^{* *}$ &, $168^{* *}$ &, $096^{*}$ &, $253^{* *}$ \\
\hline & Sig. &, 000 &, 000 &, 003 &, 001 & 049 &, 000 \\
\hline & $\mathrm{N}$ & 420 & 420 & 420 & 420 & 420 & 420 \\
\hline \multirow{3}{*}{ Control } & Rho spearman &, $189^{* *}$ & $189^{* *}$ &, $123^{*}$ & $148^{* *}$ & $175^{* *}$ & $266^{* *}$ \\
\hline & Sig. &, 000 &, 000 &, 012 & ,002 &, 000 &, 000 \\
\hline & $\mathrm{N}$ & 420 & 420 & 420 & 420 & 420 & 420 \\
\hline
\end{tabular}

$* * \mathrm{p}>0,01$

De acuerdo con los resultados de la Tabla 10 del índice de correlación rho de spearman, se observan correlaciones significativas $(\mathrm{p}>0,01)$ de nivel bajo entre factores de resiliencia y dimensiones de autoconcepto que varían de 0,123 a 0,341. Observándose un mayor índice de correlación entre la Resiliencia y la Dimensión Autoconcepto Familiar.

TABLA 11. Diferencia de medias en autoconcepto según sexo

\begin{tabular}{lcccccc}
\hline & Físico & Social & Familiar & Intelectual & Personal & Control \\
\hline $\begin{array}{l}\text { U de Mann- } \\
\text { Whitney }\end{array}$ & 17658,500 & 19169,000 & 18943,000 & 20104,500 & 21102,500 & 19211,000 \\
$\mathrm{Z}$ & $-3,397$ & $-2,174$ & $-2,358$ & $-1,416$ &,- 609 & $-2,141$ \\
$\begin{array}{l}\text { Sig. asintót. } \\
\text { (bilateral) }\end{array}$ &, 001 &, 030 &, 018 &, 157 &, 542 &, 032 \\
\hline
\end{tabular}


Según la Tabla 11, el nivel crítico bilateral (Significación asintótica bilateral) vale entre 0,001 y 0,547 . Debido a que en el caso de Autoconcepto Físico, Social, Familiar y Control son menores a 0,05 , podemos señalar que existen diferencias significativas en los promedios en grupos definidos por la variable sexo (hombre, mujer).

TABLA 12. Diferencia de medias en resiliencia según sexo

\begin{tabular}{lcccccc}
\hline & Autoestima & Empatía & Autonomía & Humor & Creatividad & Resiliencia \\
\hline $\begin{array}{l}\text { U de Mann- } \\
\text { Whitney }\end{array}$ & 17475,000 & 17714,500 & 20899,500 & 20792,000 & 19625,000 & 18477,000 \\
Z & $-3,621$ & $-3,407$ &,- 784 &,- 875 & $-1,848$ & $-2,736$ \\
$\begin{array}{l}\text { Sig. asintót. } \\
\text { (bilateral) }\end{array}$ &, 000 &, 001 &, 433 &, 381 &, 065 &, 006 \\
\hline
\end{tabular}

Según la Tabla 12, el nivel crítico bilateral (Significación asintótica bilateral) vale entre 0,000 y 0,433. En el caso de Autoestima, Empatía y Resiliencia, son menores a 0,05, podemos señalar que existen diferencias significativas en los promedios en grupos definidos por la variable sexo (hombre, mujer).

\section{DISCUSIÓN}

Partimos definiendo el autoconcepto como la percepción que el individuo tiene de sí mismo, basado en sus experiencias con los demás y en las atribuciones de su propia conducta que involucra componentes emocionales, sociales, físicos y académicos (Musitu, García y Gutiérrez, 1997), y la resiliencia como la capacidad de las personas para desarrollarse psicológicamente sanas y exitosas, a pesar de estar expuestas a situaciones adversas que amenazan su integridad (Rutter, 1993).

Ahora bien, al estudiar el autoconcepto no solo debemos entender y analizar su proceso evolutivo en la etapa escolar, sino también cómo está influido o ligado a otros aspectos del desarrollo cognitivo y social, y de qué manera la percepción que el niño tiene de sí mismo impacta su conducta y su desempeño escolar.

En la presente investigación se halló relación significativa entre la resiliencia y el autoconcepto (véase Tabla 10). Lo que nos permite afirmar que el autoconcepto determina patrones de comportamiento resiliente a los alumnos estudiados, permitiéndoles superar los problemas y dificultades del entorno. Concluiremos a partir de la presente investigación que el vínculo entre ambos procesos estudiados es relevante y se encuentra presente en la vida cotidiana escolarizada.

De acuerdo con los resultados descriptivos obtenidos sobre los factores de la resiliencia de los alumnos evaluados, se observa que la mayoría de la muestra estudiada, se ubica en el nivel medio en el puntaje general y en sus dimensiones (Véase Tabla 1); siendo la Autoestima y la Empatía las que presentan un mayor promedio, lo que está reflejando que 
los alumnos son capaces de afrontar y recuperarse de las situaciones difíciles, además de lograr comprender las dificultades de sus compañeros.

Para el caso del Autoconcepto se encontraron resultados similares, la mayoría de los alumnos se ubica en el nivel medio (Véase Tabla 2). Esto reflejaría que los alumnos evaluados en la apreciación del Autoconcepto Familiar y Físico, revelan que las vivencias y aceptación familiar así como la apreciación valorativa de las características físicas, constituyen los aspectos relevantes al hablar de Autoconcepto en estudiantes; estos resultados son similares a los obtenidos por Aguirre (2002), que señala que la familia es considerada como factor protector de la capacidad resiliente. Aguilar (2011) en este sentido afirma que existen tres posibles fuentes de factores que en su calidad de protectores, promueven comportamientos resilientes. Estos factores son los atributos personales, los apoyos del sistema familiar y aquellos provenientes de la comunidad, confirmando lo hallado en nuestra investigación. En este sentido es importante anotar que la familia resiliente facilita la formación de un autoconcepto positivo en sus hijos (Gil, 2010).

Si consideramos que las dimensiones de la resiliencia (Vanistendael, 1997), son: la existencia de redes sociales, el sentido de vida, autoestima positiva, presencia de aptitudes y destrezas y el sentido del humor, podemos establecer que la resiliencia en el caso de los sujetos de estudio se construye a partir de presentar una autoestima positiva y la existencia de redes sociales familiares. Teniendo en cuenta esto, las relaciones entre la Resiliencia y los componentes del Autoconcepto, comprobamos que la Autoestima y la Empatía correlacionan de modo significativo con todos los componentes del Autoconcepto, esto podría indicar que las experiencias positivas sobre uno mismo y en la relación con otros ejercen efecto de protección en los estudiantes, ante las dificultades y factores de riesgo; lo cual podría explicarse a partir de considerar que el procesamiento cognitivo y afectivo de las experiencias influyen en el desarrollo de la resiliencia.

Al considerar la comparación del autoconcepto y la resiliencia según el género (Véase Tablas 11 y 12), encontramos que existen diferencias significativas en las dimensiones del Autoconcepto Físico, Social, Familiar y Control, asimismo en Autoestima, Empatía y Resiliencia, en todos estos casos son las mujeres las que obtienen mayores promedios. Estos resultados son similares a los hallados por Flores (2008) al estudiar los niveles de resiliencia y el grado de definición del proyecto de vida en estudiantes, pues encuentra que existe relación entre el grado de resiliencia y el grado de definición del proyecto de vida, tanto en varones como en mujeres.

Ahora bien, la existencia en el Perú de una ideología y prácticas psicosociales de tipo sexista y las respuestas asumidas por vastos sectores del población femenina de oposición y resistencia estarían en la base de la resiliencia encontrada en la presente investigación.

\section{CONCLUSIONES}

1. El Cuestionario de Autoconcepto propuesto por Belén García Torres, presenta validez de constructo y confiabilidad por consistencia interna.

2. La resiliencia se presenta en nivel medio en general y en sus dimensiones, siendo la Autoestima y la Empatía las que presentan un mayor promedio. 
3. El Autoconcepto se sustenta básicamente en la apreciación del Autoconcepto Familiar y Físico.

4. Autoestima y la Empatía correlacionan de modo significativo con todos los componentes del Autoconcepto.

5. Al comparar a los alumnos según el sexo se encontró diferencias significativas en Autoconcepto Físico, Social, Familiar y Control, asimismo en Autoestima, Empatía y Resiliencia, en todos estos casos son las mujeres las que obtienen mayores promedios.

\section{REFERENCIAS BIBLIOGRÁFICAS}

1. Acevedo, V. y Mondragón, H. (2005). Resiliencia y escuela. Pensamiento Psicológico, 1(5), pp. 21-35.

2. Aguirre, P. (1994). Autoconcepto y dimensiones de personalidad en niños desplazados residentes en la Comunidad de Santa Cruz. (Tesis de Licenciatura no publicada). Lima, Perú: Universidad Peruana Cayetano Heredia.

3. Aguilar, L. (2011). Niños y niñas expuestos a violencia de género: Una forma de maltrato infantil. Recuperado el 13 de mayo 2011 desde http://www.malostratos. org/images/pdf/sap/Copia \% 20de\%20Exposicion\%20a \%20\%20VG\%20una \% 20 forma $\% 20 \mathrm{de} \% 20$ maltrato \%20infantil\%20LOLA \%20AGUILAR.pdf

4. Aguirre, A. (2002). Capacidad y factores asociados a la resiliencia, en adolescentes del C.E. Mariscal Andrés Avelino Cáceres del Sector IV de Pamplona Alta San Juan de Miraflores. (Tesis de Licenciatura no publicada). Lima, Perú: Facultad de Medicina Humana de la Universidad Nacional Mayor de San Marcos.

5. Astete, A.; Benavides, G. y Palacios, E. (2009). Resiliencia en niños de 8 a 12 años provenientes de familias disfuncionales. (Tesis de Licenciatura no publicada). Lima, Perú: Universidad Peruana Cayetano Heredia.

6. Badilla (2003) Badilla, L. (2003). Para comprender el concepto de Resiliencia. Recuperado el 20 de marzo de 2010, http://www.medicosenprevencion.com.ar/html/ htmldoc/trabajo/badilla.htm

7. Benard, B. (2002). From risk to resiliency: what schools can do. Portland, O. R.: Western Center for Drug-Free Schools and Communities.

8. Caravedo, V (2001). Autoconcepto en hijas de padres separados: Un estudio descriptivo (Tesis de Licenciatura no publicada). Lima, Perú: Universidad Peruana Cayetano Heredia.

9. Epstein, N (1990). Identificación de los factores educativos de un modelo de resiliencia que los padres estén activamente involucrados. Recuperado el 11 de febrero de 2010 , www.human.ula.ve/doctoradoeneducacion/documentos/anzola.pdf

10. Flores, M. (2008). Resiliencia y proyecto de vida en estudiantes del tercer año de secundaria de la UGEL 03. (Tesis de Licenciatura no publicada). Lima, Perú: Universidad Nacional Mayor de San Marcos. 
11. Gil, G. (2010). Los procesos holísticos de resiliencia en el desarrollo de identidades autorreferenciadas en lesbianas, gays y bisexuales. (Tesis Doctoral no publicada). Islas Canarias, España: Universidad de Las Palmas de Gran Canaria.

12. Kalawski, J. y Haz, A. (2003). ¿Dónde está la resiliencia? Una Reflexión Conceptual. Interamerican Journal of Psychology, 37(2), pp. 365-372.

13. Loperena, M. (2008). El autoconcepto en niños de cuatro a seis años. Tiempo de Educar, 9(18), pp. 307-327.

14. Mori, P. (1989). Estudio del autoconcepto en niños y niñas de niveles socioeconómicos medio y bajo de dos centros educativos de Lima. (Tesis de Maestría no publicada). Lima, Perú: Universidad Peruana Cayetano Heredia.

15. Mori, P. (2002). Personalidad, autoconcepto y percepción del compromiso parental: Sus relaciones con el rendimiento académico en alumnos del sexto grado. (Tesis de Doctoral no publicada). Lima, Perú: Universidad Nacional Mayor de San Marcos.

16. Musitu, G., García, F. \& Gutiérrez, M. (1997). Autoconcepto Forma A (A.F.A). Manual. Madrid: TEA Ediciones.

17. Orbegoso, D. (2002). Autoconcepto y depresión en niños obesos y no obesos. (Tesis de Maestría no publicada). Lima, Perú: Universidad Peruana Cayetano Heredia.

18. Pazzaglia, P. (1990). Relaciones entre el autoconcepto y las preferencias personales en alumnos de secundaria del Colegio Parroquial Mixto "Fe y Alegría N. 5 " Chacarilla de Otero. (Tesis de Bachiller no publicada). Lima, Perú: Universidad Peruana Cayetano Heredia.

19. Rutter, M. (1993). Resilience: Some conceptual considerations. Journal of Adolescent Health, 14, pp. 626-631.

20. Rutter, M. (1999). Resilience concepts and findings: implications for family therapy. Journal of Family Therapy, 21, pp. 119-144.

21. Salgado, A.C. (2005). Métodos para medir la resiliencia: Una alternativa peruana. Liberalit, Revista de Psicología, 11, pp. 41-48.

22. Suárez, E (1995). Resiliencia y aprendizaje en sectores populares. Recuperado 30 de abril de 2010, www.rieoei.org/deloslectores/1466Szarazgat.pdf

23. Suxe, G. (2004). Relación entre autoconcepto, actitudes maternas y variables demográficas en niños de 6 y 7 años. (Tesis de Licenciatura no publicada). Lima, Perú: Universidad Peruana Cayetano Heredia.

24. Urquijo, S. (2002). Auto-concepto y desempeño académico en adolescentes. Relaciones con sexo, edad e institución. Psico-USF, 7(2), pp. 211-218.

25. Vanistendael, S. (1997). Resiliencia. Conferencia presentada en el Seminario: Los aportes del concepto de resiliencia en los programas de intervención psicosocial. Pontificia Universidad Católica de Chile. Santiago, Chile. 
FACTORES PERSONALES DE RESILIENCIA Y AUTOCONCEPTO EN ESTUDIANTES DE PRIMARIA DE LIMA METROPOLITANA

ANEXO. Percentiles de autoconcepto. Según puntaje directo

\begin{tabular}{|c|c|c|c|c|c|c|}
\hline Percentil & Físico & Social & Familiar & Intelectual & Personal & Control \\
\hline 1 & 13 & 13 & 16 & 15 & 14 & 12 \\
\hline 2 & 15 & 15 & 18 & 16 & 16 & 15 \\
\hline 3 & 16 & 16 & 19 & & 17 & 16 \\
\hline 4 & 17 & 17 & 20 & 17 & 18 & 17 \\
\hline 5 & 18 & & 21 & & & \\
\hline 6 & & 18 & & & 19 & \\
\hline 7 & 19 & & & 18 & & 18 \\
\hline 8 & & & 22 & & 20 & 19 \\
\hline 9 & & 19 & 23 & & & \\
\hline 10 & 20 & 20 & & 19 & 21 & \\
\hline \multicolumn{7}{|l|}{11} \\
\hline 12 & 21 & & & & 22 & \\
\hline 13 & & 21 & & & & 20 \\
\hline 14 & & & & 20 & & \\
\hline 15 & 22 & & 24 & & & \\
\hline 16 & & 22 & & & & \\
\hline 17 & & & & & 23 & \\
\hline 18 & & & 25 & & & 21 \\
\hline 19 & 23 & & & 21 & & \\
\hline 21 & & & & & 24 & \\
\hline 23 & & & & & & 22 \\
\hline 24 & & 23 & & & & \\
\hline 25 & 24 & & & & & \\
\hline 26 & & & 26 & 22 & 25 & \\
\hline 29 & 25 & 24 & & & & \\
\hline 31 & & & & 23 & & 23 \\
\hline 32 & & & & & 26 & \\
\hline 33 & 26 & & & & & \\
\hline 34 & & 25 & & & & \\
\hline 35 & & & & 24 & & \\
\hline 38 & 27 & & & & 27 & \\
\hline 39 & & & & & & 24 \\
\hline 40 & & & & 25 & & \\
\hline 42 & 28 & & & & & \\
\hline 43 & & & 30 & & & \\
\hline 44 & & & & & 28 & \\
\hline 45 & & & & 26 & & \\
\hline 46 & & 27 & & & & 25 \\
\hline 47 & 29 & & & & & \\
\hline 49 & & & & 27 & & \\
\hline 51 & & 28 & 31 & & & \\
\hline 53 & & & & & 29 & 26 \\
\hline 54 & 30 & & & & & \\
\hline 55 & & & & 28 & & \\
\hline 58 & & 29 & 32 & & & \\
\hline 59 & 31 & & & & & 27 \\
\hline
\end{tabular}




\begin{tabular}{|c|c|c|c|c|c|c|}
\hline Percentil & Físico & Social & Familiar & Intelectual & Personal & Control \\
\hline 60 & & & & 29 & & \\
\hline 61 & & & & & 30 & \\
\hline 64 & 32 & & & & & \\
\hline 65 & & 30 & & 30 & & \\
\hline 66 & & & 33 & & & \\
\hline 67 & & & & & & 28 \\
\hline 69 & & & & 31 & & \\
\hline 70 & 33 & 31 & & & 31 & \\
\hline 72 & & & 34 & & & 29 \\
\hline 73 & & & & 32 & & \\
\hline 74 & 34 & & & & & \\
\hline 76 & & 32 & & & & \\
\hline 77 & 35 & & & 33 & & 30 \\
\hline 78 & & & 35 & & 32 & \\
\hline 79 & 36 & & & & & \\
\hline 80 & & 33 & & & & \\
\hline 83 & & & & 34 & & \\
\hline 84 & & & & & & 31 \\
\hline 85 & 37 & 34 & & & & \\
\hline 86 & & & & & 33 & \\
\hline 87 & & & 36 & 35 & & \\
\hline 89 & 38 & & & & & 32 \\
\hline 90 & & & 37 & & & \\
\hline 91 & 39 & & & 36 & & 33 \\
\hline 93 & & & 38 & & & \\
\hline 94 & & & & 37 & & 34 \\
\hline 95 & & 37 & & 38 & 34 & \\
\hline 96 & & 38 & 39 & & & \\
\hline 97 & & & & 39 & & 35 \\
\hline 98 & & 39 & & & & 36 \\
\hline 99 & 40 & 40 & 40 & 40 & 35 & 37 \\
\hline
\end{tabular}

